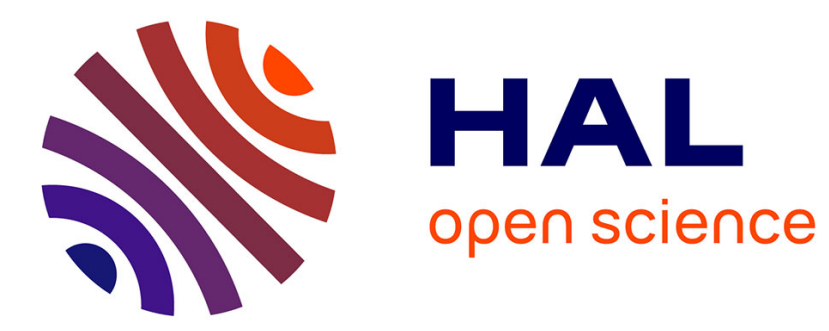

\title{
A multiple data secure communication based on synchronization of chaotic systems with zero dynamics
}

Gang Zheng, Driss Boutat, Latifa Boutat-Baddas, Thierry Floquet, Jean-Pierre Barbot

\section{- To cite this version:}

Gang Zheng, Driss Boutat, Latifa Boutat-Baddas, Thierry Floquet, Jean-Pierre Barbot. A multiple data secure communication based on synchronization of chaotic systems with zero dynamics. ECC'07 - European Control Conference, Jul 2007, Kos, Greece. pp.5878-5883. inria-00193215

\section{HAL Id: inria-00193215 https://hal.inria.fr/inria-00193215}

Submitted on 2 Dec 2007

HAL is a multi-disciplinary open access archive for the deposit and dissemination of scientific research documents, whether they are published or not. The documents may come from teaching and research institutions in France or abroad, or from public or private research centers.
L'archive ouverte pluridisciplinaire HAL, est destinée au dépôt et à la diffusion de documents scientifiques de niveau recherche, publiés ou non, émanant des établissements d'enseignement et de recherche français ou étrangers, des laboratoires publics ou privés. 


\title{
A Multiple Data Secure Communication Based on Synchronization of Chaotic Systems with Zero Dynamics
}

\author{
G. Zheng, D. Boutat, L. Boutat-Baddas, T. Floquet and J.P. Barbot
}

\begin{abstract}
This paper gives a new scheme of data secure communication based on synchronization of chaotic systems with multi inputs multi outputs and zero dynamics. In addition, a sliding mode observer is proposed and a simple example and simulations are given in order to highlight the proposed method.
\end{abstract}

\section{INTRODUCTION}

Since chaotic system is extremely sensitive to initial conditions and parameters variations, its application to secure communication has provoked a great deal of interest, especially after Carroll and Pecora's outstanding work on successfully synchronizing two chaotic systems [8].

Up to now, most chaos-based communication systems are based on chaos synchronization technique. Actually, this synchronization can be studied as an observer design problem, since the work of $\mathrm{H}$. Nijmeijer and I. Mareels in [7].

In secure communication system, we need to estimate not only the state for chaos synchronization but also the input (confidential message), which can be considered as to design an unknown inputs observer with unknown inputs estimation. For the linear systems with unknown inputs and without input estimation, in [4], [5], the necessary and sufficient conditions for the existence of a classical observer are presented. Moreover, an algorithms of full order observers and conditions for their existence are stated in [2]. In the nonlinear case, at our knowledge, one of the first design of unknown inputs observer was given in [11] where authors relaxed the Usual Observability Matching condition. Roughly speaking the UOM condition means that all unknown inputs act on the first derivative of the output and also verify regularity properties. In [3] for the linear case and in [1] for nonlinear case a new observability matching condition are given, which relaxed again the previously mentioned conditions. Moreover, in the last reference the authors give conditions in order to recover the unknown

G. Zheng is with INRIA Rhône-Alpes, 38330 Montbonnot Saint Ismier, France. gang. zhengeinria.fr

D.Boutat is with LVR, ENSI-Bourges, Université d'Orléans, 10, Bd. Lahitolle, 18020 Bourges Cedex, France. driss.boutateensi-bourges. fr

L. Boutat-Baddas is with UHP Nancy I, CRAN-UMR 7039CNRS-INPL-UHP, IUT de Longwy, 54400 Cosnes-et-Romain, France. latifa.boutat-baddaseiut-longwy. uhp-nancy. fr

T. Floquet is with LAGIS UMR CNRS 8146, Ecole Centrale de Lille, BP 48, Cité Scientifique, 59651 Villeneuve-d'Ascq and Project ALIEN, INRIAFutur, France. thierry.floqueteec-lille.fr

J.P. Barbot is with Equipe Commande des Systèmes (ECS), ENSEA, 6 Av. du Ponceau, 95014 Cergy Cedex and Project ALIEN, INRIA-Futur, France. barboteensea.fr inputs for systems without zero dynamics. This problem can be see as a Left Invertibility Problem [10].

In this paper, we propose to apply the results of [1] taking into account the stability of the zero dynamics into multi data secure communication scheme.

This paper is organized as follows: Notations and problem statement are presented in section 2 . In section 3, a new scheme of transmitter based on the stability of zero dynamics is proposed. Moreover, A sliding mode observer design with unknown inputs estimation (receiver) is proposed in section 4. Finally, in order to highlight the well found of the proposed method, an example is given simulated and commented.

\section{NOTATIONS AND PROBLEM STATEMENT}

A multiple secure communication system can be represented in the following form:

$$
\left\{\begin{array}{l}
\dot{x}=f(x)+\sum_{i=1}^{m} g_{i}(x) u_{i} \\
y=\left[h_{1}(x), \ldots, h_{p}(x)\right]^{T}
\end{array}\right.
$$

where $U$ is an open set of $R^{n}, x \in U$ is the state vector and $f: R^{n} \rightarrow R^{n}$ is analytic. $y \in R^{p}$ is the output vector and $u \in R^{m}$ represents the confidential information to be transmitted. The vector fields $f=\left[f_{1}, \ldots, f_{n}\right]^{T}, g_{i}=$ $\left[g_{i 1}, \ldots, g_{i m}\right]^{T}$ and $h=\left[h_{1}, \ldots, h_{p}\right]^{T}$ are assumed to be sufficiently smooth on $U$, where $f_{i}, h_{j} \in R$ and $g_{k} \in R^{n}$, $i \in[1, n], j \in[1, p], k \in[1, m]$. Without loss of generality, it is assumed that the distribution $\operatorname{span}\left\{g_{1}, \ldots, g_{m}\right\}$ and the codistribution $\operatorname{span}\left\{d h_{1}, \ldots, d h_{p}\right\}$ are nonsingular on $U$.

The relative degree $r$ of system (1) is defined by $r=$ $\left\{r_{1}, \ldots, r_{p}\right\}$, where $r_{i}=\min \left\{s\right.$ such that $L_{g_{k}} L_{f}^{s-1} h_{i} \neq 0$ for $k=1: m\}, i=1: p$.

Under the case $r=\sum_{i=1}^{p} r_{i}=n$, a multiple secure communication system can be established easily. However, this method does not lead to an enough complexity of computation for decryption. Therefore in order to improve the security, the complexity of the scheme can be increased by choosing the outputs $y$ and the input channel vectors $\left\{g_{1}, \ldots, g_{m}\right\}$ such that $r<n$. In [1], an algorithm to solve the observation problem for nonlinear systems with unknown inputs when $r<n$ has been proposed.

It is assumed that $p \geq m$, and that the system (1) has a relative degree $r=\left\{r_{1}, \ldots, r_{p}\right\}$. It should be noted that in this paper we do not consider the case of infinite relative degree. Let us define the following sets that will be used in the sequel: 
- $\Omega=\left\{d h_{1}, \ldots, d L_{f}^{r_{1}-1} h_{1}, \ldots, d h_{p}, \ldots, d L_{f}^{r_{p}-1} h_{p}\right\}$ and $£$ is the related distribution:

$$
£=\operatorname{span}\left\{h_{1}, \ldots, L_{f}^{r_{1}-1} h_{1}, \ldots, h_{p}, \ldots, L_{f}^{r_{p}-1} h_{p}\right\}
$$

where $r=\operatorname{dim} \Omega=\sum_{i=1}^{p} r_{i}$.

- $G$ is the smallest involutive distribution that contains $\left\{g_{1}(x), \ldots, g_{m}(x)\right\}$. Note $k=\operatorname{dim} G, m \leq k \leq n$.

- $G^{\perp}$ is the annihilator of $G$ :

$G^{\perp}=\operatorname{span}\left\{\alpha_{1}, \ldots, \alpha_{n-k}\right\}$, where the $\alpha_{i}$ are one-forms such that for all $\lambda \in G, l_{\lambda} \alpha_{i}=0$ for $i=1: n-k$, where $l_{\lambda} \alpha=\alpha(\lambda)$ is the inner product of the vector field $\lambda$ and $\alpha$.

Assume $r<n$. There exists a transformation $(\xi, \eta)=$ $\phi(x)$ such that the system (1) can be locally transformed into the following normal form:

$$
\left\{\begin{array}{l}
\dot{\xi}_{1}^{i}=\xi_{2}^{i} \\
\vdots \\
\dot{\xi}_{r_{i}-1}^{i}=\xi_{r_{i}}^{i} \\
\dot{\xi}_{r_{i}}^{i}=L_{f}^{r_{i}} h_{i}(x)+\sum_{j=1}^{m} L_{g_{j}} L_{f}^{r_{i}-1} h_{i}(x) u_{j} \\
\dot{\eta}=Q(\xi, \eta, u) \\
y_{i}=\xi_{1}^{i}
\end{array}\right.
$$

where $\xi=\left[\begin{array}{lll}\xi^{1} & \cdots & \xi^{p}\end{array}\right]^{T}$ and

$$
\xi^{i}=\left[\begin{array}{c}
\xi_{1}^{i} \\
\vdots \\
\xi_{r_{i}}^{i}
\end{array}\right]=\left[\begin{array}{c}
h_{i}(x) \\
\vdots \\
L_{f}^{r_{i}-1} h_{i}(x)
\end{array}\right], \text { for } i \in[1, p] .
$$

If $r<n$ and if the distribution $\operatorname{span}\left\{g_{1}, \ldots, g_{m}\right\}$ is not involutive, $u$ can not be obtained using classical observation algorithm. [1] provided an algorithm, under sufficient conditions, to allow the recovery of both the state and the unknown inputs in finite time. The main idea of this algorithm is to find extra information through functions of the outputs and their time derivatives. Let us define:

$$
\begin{aligned}
V & =\left[\begin{array}{lll}
L_{f}^{r_{1}} h_{1}(x) & \cdots & L_{f}^{r_{p}} h_{p}(x)
\end{array}\right]^{T}+\Gamma(x) u \\
& =\left[\begin{array}{lll}
y_{1}^{\left(r_{1}\right)} & \cdots & y_{p}^{\left(r_{p}\right)}
\end{array}\right]^{T}
\end{aligned}
$$

that can be known using the normal form (2).

Assume there exist $1 \times p$ vector functions

$$
K(x)=\left[k_{1}(x), \ldots, k_{p}(x)\right] \in £(x), \text { with } K(x) \neq 0
$$

such that

$$
K \Gamma=0
$$

and define a fictitious output as follows:

$$
\bar{y}=\bar{h}(x)=K V=\sum_{i=1}^{p} k_{i}(x) L_{f}^{r_{i}} h_{i}(x) .
$$

If $\bar{y} \notin £(x)$, it can be considered as a suitable fictitious output in order to estimate more states ${ }^{1}$. Set $y=[y, \bar{y}]^{T}$. The

\footnotetext{
${ }^{1}$ It should be noticed that there may exist a submanifold of singularity $S=\{x \in U$ such that $\bar{h}(x) \in £(x)\}$.
}

system has a new relative degree with respect to this output and the algorithm iterates until the new relative degree with all new fictitious outputs is equal to $n$, and then it has been shown in [1] that both the state $x$ and the unknown input $u$ can be estimated in finite time. And a more general case: $r<$ $n$ (even with all new fictitious outputs $y$ ), will be discussed in this paper. In the next section, several hypothesis will be given for system (2) in order to deduce a practical form with zero dynamics, which can be applied into multi data secure communication based on synchronization of chaotic system.

\section{ZERO DYNAMICS}

Suppose that after $s^{t h}$ steps iteration, all new fictitious outputs construct the $\bar{£}(x)$ as follows:

$$
\begin{aligned}
\bar{£}(x)= & \operatorname{span}\left\{h_{1}, \ldots, L_{f}^{r_{1}-1} h_{1}, \ldots, h_{p}, \ldots, L_{f}^{r_{p}-1} h_{p},\right. \\
& \left.\bar{y}_{p+1}, \ldots, L_{f}^{r_{p+1}-1} \bar{y}_{p+1}, \ldots \bar{y}_{p+s}, \ldots, L_{f}^{r_{p+s}-1} \bar{y}_{p+s}\right\}
\end{aligned}
$$

and if $\operatorname{dim} \bar{£}=n$, then we can recover all the states and all the unknown inputs. However, for the last time iteration of this algorithm, if there is no new $\bar{y} \notin \overline{\mathscr{E}}$, or if there is a singularity, moreover if $\operatorname{dim} \bar{£}<n$, can we still recover all the states and all the unknown inputs? The answer is based on the Proposition 1.3 in Chapter 5 of [6]:

Proposition 1: [6]If $\operatorname{dim} G=k=m$, which means that the distribution spanned by $\left\{g_{1}(x), \ldots, g_{m}(x)\right\}$ is involutive, then $\operatorname{dim} G^{\perp}=n-m$. Moreover if $\operatorname{dim}\left(G^{\perp} \cup \Omega\right)=n$, where

$$
\Omega=\left\{d h_{1}, \ldots, d L_{f}^{r_{1}-1} h_{1}, \ldots, d h_{m}, \ldots, d L_{f}^{r_{m}-1} h_{m}\right\}
$$

then there exists a diffeomorphism which can transform the original system into the following form with zero dynamics:

$$
\left\{\begin{array}{l}
\dot{\xi}_{1}^{i}=\xi_{2}^{i} \\
\vdots \\
\dot{\xi}_{r_{i}-1}^{i}=\xi_{r_{i}}^{i} \\
\dot{\xi}_{r_{i}}^{i}=a_{i}(\xi, \eta)+\sum_{j=1}^{m} b_{j}^{i}(\xi, \eta) u_{j} \\
\dot{\eta}=Q(\xi, \eta) \\
y_{i}=\xi_{1}^{i}
\end{array}\right.
$$

where $a_{i}(\xi, \eta)=L_{f}^{r_{i}} h_{i \mid \phi^{-1}(\xi, \eta)}$, and $b_{j}^{i}(\xi, \eta)=$ $L_{g_{j}} L_{f}^{r_{i}-1} h_{i \mid \phi^{-1}(\xi, \eta)}$, for $i \in[1, p]$

Remark 1: For system (1), if $p=m=1$, there is only one input $g$, which is obviously involutive. So in this case, it can be also transformed into the system (7).

Moreover, the following lemma is an extension of the above proposition for the case where the inputs are not involutive.

Lemma 1: Suppose that the last iteration of the algorithm gives $\bar{£}(x)$ defined in (6) where $\operatorname{dim} \bar{£}=q<n$ and $\operatorname{rank} \Gamma=m$. Define

$$
\begin{aligned}
\Omega= & \left\{d h_{1}, \ldots, d L_{f}^{r_{1}-1} h_{1}, \ldots, d h_{m}, \ldots, d L_{f}^{r_{m}-1} h_{m}, d \bar{y}_{p+1},\right. \\
& \left.\ldots, d L_{f}^{r_{p+1}-1} \bar{y}_{p+1}, \ldots, d \bar{y}_{p+s}, \ldots, d L_{f}^{r_{p+s}-1} \bar{y}_{p+s}\right\}
\end{aligned}
$$


If

$$
\operatorname{dim}\left(G^{\perp} \cup \Omega\right)=n
$$

then system (2) can be described into form (7), where $a_{i}(\xi, \eta)=L_{f}^{r_{i}} h_{i \mid \phi^{-1}(\xi, \eta)}$, and $b_{j}^{i}(\xi, \eta)=$ $L_{g_{j}} L_{f}^{r_{i}-1} h_{i \mid \phi^{-1}(\xi, \eta)}$, for $i \in[1, p+s]$.

Proof: Suppose that

$$
\operatorname{dim}\left(G^{\perp} \cup \Omega\right)=n
$$

with $\operatorname{dim} \bar{£}=q<n$ and $\operatorname{rank} \Gamma=m$, it is possible to find $(n-q)$ functions from the set $\left\{\lambda_{1}, \ldots, \lambda_{n-k}\right\}$, without loss of generality, choosing $\left\{\lambda_{1}, \ldots, \lambda_{n-q}\right\}$, such that, the $n$ differentials $\left\{d h_{1}, \ldots, d L_{f}^{r_{1}-1} h_{1}, \ldots, d h_{p}, \ldots, d L_{f}^{r_{p}-1} h_{p}\right.$,

$$
d \bar{y}_{p+1}, \ldots, d L_{f}^{r_{p+1}-1} \bar{y}_{p+1}, \ldots, d \bar{y}_{p+s}, \ldots, d L_{f}^{r_{p+s}-1} \bar{y}_{p+s},
$$

$\left.d \lambda_{1}, \ldots, d \lambda_{n-q}\right\}$ are linearly independent. Then there exists a diffeomorphism

$$
\begin{aligned}
\phi= & \left\{h_{1}, \ldots, L_{f}^{r_{1}-1} h_{1}, \ldots, h_{p}, \ldots, L_{f}^{r_{p}-1} h_{p},\right. \\
& \bar{y}_{p+1}, \ldots, L_{f}^{r_{p+1}-1} \bar{y}_{p+1}, \ldots, \bar{y}_{p+s}, \ldots, \\
& \left.L_{f}^{r_{p+s}-1} \bar{y}_{p+s}, \lambda_{1}, \ldots, \lambda_{n-q}\right\}
\end{aligned}
$$

which transforms system (2) into the form (7), whose zero dynamics part is given by

$$
\dot{\eta}=Q(\xi, \eta)_{\mid \xi=0}
$$

\section{Remarks 1:}

i) If $\operatorname{dim} \bar{£}=r<n$ and $\operatorname{dim}\left(G^{\perp} \cup \Omega\right)<n$, then there does exist zero dynamics part in the transformed system, i.e., equation (10) becomes $\dot{\eta}=Q(\xi, \eta, u)$.

ii) If for all $i \in[1, p+s], j \in[1, n-q], \frac{\partial \dot{\xi}_{r_{i}}^{i}}{\partial \eta_{1}}=\ldots=$ $\frac{\partial \dot{\xi}_{r_{i}}^{i}}{\partial \eta_{n-q}}=0$, i.e., equation (10) becomes $\dot{\eta}=Q(\eta)$, then it is possible to recover the unknown input $u$.

iii) If for a certain $i \in[1, p+s], j \in[1, n-q]$, we have $\frac{\partial \dot{\xi}_{r_{i}}^{i}}{\partial \eta_{j}} \neq 0$, then the unknown input $u$ depends on the detectability of $\eta$.

For solving the problem stated above, we need the following hypothesis:

Hypothesis 1: The zero dynamics of system (7) is uniformly, at least exponentially detectable, i.e., there exists a strictly definitive positive Lyapunov function $V(\eta-\bar{\eta})$ which satisfies, for all $\eta, \bar{\eta}$ and $\xi$, the following relations:

$$
\dot{V}(\eta-\bar{\eta})=\frac{\partial V}{\partial(\eta-\bar{\eta})}[Q(\xi, \eta)-Q(\xi, \bar{\eta})] \leqslant-K_{0} V
$$

where $K_{0}$ is a positive constant. And moreover $\phi$ defined in (9) is a diffeomorphism on $U$.

Hypothesis 2: The system (1) is Bounded Input Bounded State (BIBS).

In the next section, for the proposed system (7), a sliding mode observer is designed in order to recover both state and unknown inputs.

\section{OBSERVER DESIGN WITH UNKNOWN INPUTS ESTIMATION}

Under the Hypothesis 1 and 2, we can observe both state and unknown inputs of system (7) using the following sliding mode observer

$$
\left\{\begin{array}{l}
\dot{\hat{\xi}}_{1}^{i}=\hat{\xi}_{2}^{i}+\lambda_{1}^{i} \operatorname{sign}\left(\xi_{1}^{i}-\hat{\xi}_{1}^{i}\right) \\
\dot{\hat{\xi}}_{2}=\hat{\xi}_{3}^{i}+E_{1}^{i} \lambda_{2}^{i} \operatorname{sign}\left(\tilde{\xi}_{2}^{i}-\hat{\xi}_{2}^{i}\right) \\
\vdots \\
{ }_{i} \\
\hat{\xi}_{r_{i}-1}=\hat{\xi}_{r_{i}}^{i}+E_{r_{i}-2}^{i} \lambda_{r_{i}-1}^{i} \operatorname{sign}\left(\tilde{\xi}_{r_{i}-1}^{i}-\hat{\xi}_{r_{i}-1}^{i}\right) \\
\cdot \hat{\hat{\xi}}_{r_{i}}=a_{i}(\tilde{\xi}, \hat{\eta})+E_{r_{i}-1}^{i} \lambda_{r_{i}}^{i} \operatorname{sign}\left(\tilde{\xi}_{r_{i}-1}^{i}-\hat{\xi}_{r_{i}-1}^{i}\right) \\
\dot{\hat{\eta}}=Q(\tilde{\xi}, \hat{\eta}) E_{n-q}
\end{array}\right.
$$

with the auxiliary states:

$$
\tilde{\xi}_{t+1}^{i}=\hat{\xi}_{t+1}^{i}+E_{t}^{i} \lambda_{t+1}^{i} \operatorname{sign}\left(\tilde{\xi}_{t+1}^{i}-\hat{\xi}_{t+1}^{i}\right)
$$

where

$$
\begin{aligned}
& E_{t}^{i}= \begin{cases}1 & \text { if } \tilde{\xi}_{t+1}^{i}=\hat{\xi}_{t+1}^{i} \text { and } E_{t-1}^{i}=1 \\
0 & \text { otherwise }\end{cases} \\
& E_{n-q}= \begin{cases}1 & \text { if all } E_{t}^{i}=1 \\
0 & \text { otherwise }\end{cases}
\end{aligned}
$$

for $i \in[1, q]$ (Noted: $\left.\tilde{\xi}_{1}^{i}=\xi_{1}^{i}\right)$.

Lemma 2: Thanks to the observer given in (11), for any initial condition, there exists $\lambda_{i}^{j}$ and $t_{r}>0$ such that $\forall t>t_{r}$, $\hat{\xi}(t)=\tilde{\xi}(t)=\xi(t)$ and $\lim _{t \rightarrow+\infty}|\eta(t)-\hat{\eta}(t)|=0$.

Proof: Set $e_{t}^{i}=\xi_{t}^{i}-\hat{\xi}_{t}^{i}$, for $i \in[1, p+s], t \in\left[1, r_{i}\right]$, $\left(\tilde{\xi}_{1}=\xi_{1}\right)$.

Assume that after the first $(j-1)^{t h}$ step, $j \in[2, p+s]$, we have obtained:

$$
\tilde{\xi}_{j-1}^{i}=\hat{\xi}_{j-1}^{i}
$$

and $E_{j-1}^{i}=1$. Then we have

$$
\tilde{\xi}_{j}^{i}=\hat{\xi}_{j}^{i}+\lambda_{j-1}^{i} \operatorname{sign}\left(\tilde{\xi}_{j-1}^{i}-\hat{\xi}_{j-1}^{i}\right)=\xi_{j}^{i} .
$$

So for the $j^{t h}$ step, the dynamic of observation error can be described in the following form:

$$
\dot{e}_{j}^{i}=\xi_{j+1}^{i}-\hat{\xi}_{j+1}^{i}-E_{j-1}^{i} \lambda_{j}^{i} \operatorname{sign}\left(\tilde{\xi}_{j}^{i}-\hat{\xi}_{j}^{i}\right)
$$

We can choose the following Lyapunov function:

$$
V_{j}^{i}=\frac{1}{2}\left(e_{j}^{i}\right)^{2}
$$

Thanks to $E_{j-1}^{i}=1$, and $\tilde{\xi}_{j}^{i}=\xi_{j}^{i}$, we have:

$$
\dot{V}_{j}^{i}=e_{j}^{i}\left[\xi_{j+1}^{i}-\hat{\xi}_{j+1}^{i}-\lambda_{j}^{i} \operatorname{sign}\left(e_{j}^{i}\right)\right]
$$

So if $\lambda_{j}^{i}>\left.\left|\xi_{j+1}^{i}-\hat{\xi}_{j+1}^{i}\right|\right|_{\max }$ (i.e., $\exists k_{j}^{i}>0$, such that $\lambda_{j}^{i}=$ $\left.k_{j}^{i}+\left|\xi_{j+1}^{i}-\hat{\xi}_{j+1}^{i}\right|_{\max }\right)$, then

$$
\dot{V}_{j}^{i}=-k|e|=-k V^{1 / 2}
$$


which means there exists $t_{j}^{i}$, such that if $t>t_{j}^{i}>t_{j-1}^{i}$, $e_{j}^{i}=\dot{e}_{j}^{i}=0$, and we obtain:

$$
\xi_{j+1}^{i}-\hat{\xi}_{j+1}^{i}=\lambda_{j}^{i} \operatorname{sign}\left(e_{j}^{i}\right)
$$

According to the definitions of (12) and (13), we have:

$$
\tilde{\xi}_{j+1}^{i}=\hat{\xi}_{j+1}^{i}
$$

and $E_{j}^{i}=1$.

Therefore, step by step we can recover all the components of vector $\xi$. But due to the unknown input $u_{i}$, we cannot recover $\eta$ through $\dot{\xi}_{r}^{i}$. However, under the Hypothesis 1 , we can reconstruct the remain states as follows.

Under the Hypothesis 2, as the state is bounded for all $t>0$, then $\eta$ is bounded. Moreover the estimation error of the zero dynamics $\left(e_{n-q}=\eta-\hat{\eta}\right)$ is equal to:

$$
\dot{e}_{n-q}=Q(\xi, \eta)-Q(\tilde{\xi}, \hat{\eta})
$$

Thus from Hypothesis 1, we deduce that

$$
\lim _{t \rightarrow \infty}|\eta(t)-\hat{\eta}(t)|=0 .
$$

Proposition 2: Under Hypothesis 1 and 2, with $\operatorname{rank} \Gamma=$ $m$, thanks to the observer (11), for any given bounded initial condition, there exists $t_{r}>0$ such that $\forall t>t_{r}$

$$
\begin{aligned}
& \hat{\xi}(t)=\tilde{\xi}(t)=\xi(t), \\
& \lim _{t \rightarrow+\infty}|\eta(t)-\hat{\eta}(t)|=0 \quad \text { and } \\
& \lim _{t \rightarrow+\infty}|u(t)-\tilde{u}(t)|=0
\end{aligned}
$$

\section{Proof:}

\section{Reconstruction of the unknown input $u$}

As $\xi$ and $u$ are bounded, we can choose

$$
\lambda_{k_{i}}^{i}>\left|a_{i}(\xi, \eta)-a_{i}(\tilde{\xi}, \hat{\eta})+\sum_{j=1}^{m} b_{j}^{i}(\xi, \eta) u_{j}\right|_{\max },
$$

such that $e_{r_{i}}^{i}$ converges to zero in finite time. Consequently we obtain $\xi=\tilde{\xi}$.

Set

$$
\Gamma=\left[\begin{array}{ccc}
b_{1}^{1}(\xi, \eta) & \cdots & b_{m}^{1}(\xi, \eta) \\
\vdots & \ddots & \vdots \\
b_{1}^{p+s}(\xi, \eta) & \cdots & b_{m}^{p+s}(\xi, \eta)
\end{array}\right]
$$

As $\operatorname{rank} \Gamma=m$, without loss of generality, we can assume that the first $m$ rows are independent, i.e., $\operatorname{rank} B(\xi, \eta)=m$, where $B(\xi, \eta)=\left[\begin{array}{ccc}b_{1}^{1}(\xi, \eta) & \cdots & b_{m}^{1}(\xi, \eta) \\ \vdots & \ddots & \vdots \\ b_{1}^{m}(\xi, \eta) & \cdots & b_{m}^{m}(\xi, \eta)\end{array}\right]$. So we have $\dot{\hat{\xi}}_{r}=A(\tilde{\xi}, \hat{\eta})+B(\tilde{\xi}, \hat{\eta}) u$, where $\dot{\hat{\xi}}_{r}=\left[\begin{array}{c}\dot{\hat{\xi}}_{r_{1}}^{1} \\ \vdots \\ \dot{\hat{\xi}} \\ \hat{\xi}_{r_{m}}\end{array}\right]$, $A(\tilde{\xi}, \hat{\eta})=\left[\begin{array}{c}a_{1}(\tilde{\xi}, \hat{\eta}) \\ \vdots \\ a_{m}(\tilde{\xi}, \hat{\eta})\end{array}\right]$ and $u=\left[\begin{array}{c}u_{1} \\ \vdots \\ u_{m}\end{array}\right]$.

Once all the states $\xi$ are observed and $\eta$ is estimated exponentially, there exists $t_{r}>t_{j}^{i}$, for all $i \in[1, p+s]$, $j \in\left[1, r_{i}\right]$, when $t>t_{r}$, such that

$$
e_{A}:=A(\xi, \eta)-A(\tilde{\xi}, \hat{\eta})
$$

then we can obtain

$$
e_{A}+B(\tilde{\xi}, \hat{\eta}) u=E_{r-1} \lambda_{r} \operatorname{sign}\left(\tilde{\xi}_{r}-\hat{\xi}_{r}\right)
$$

where $E_{r-1}=\operatorname{diag}\left\{E_{r_{1}-1}^{1}, E_{r_{2}-1}^{2}, \ldots, E_{r_{m}-1}^{m}\right\}$, $\lambda_{r}=\operatorname{diag}\left\{\lambda_{r_{1}}^{1}, \lambda_{r_{2}}^{2}, \ldots, \lambda_{r_{m}}^{m}\right\}$ and $\operatorname{sign}\left(\tilde{\xi}_{r}-\hat{\xi}_{r}\right)=$

$\left[\begin{array}{c}\operatorname{sign}\left(\tilde{\xi}_{r_{1}}^{1}-\hat{\xi}_{r_{1}}^{1}\right) \\ \vdots \\ \operatorname{sign}\left(\tilde{\xi}_{r_{m}}^{m}-\hat{\xi}_{r_{m}}^{m}\right)\end{array}\right]$.

$$
\tilde{u}=B^{-1}(\tilde{\xi}, \hat{\eta}) E_{r-1} \lambda_{r} \operatorname{sign}\left(\tilde{\xi}_{r}-\hat{\xi}_{r}\right)
$$

for $t>t_{r}$, we have

$$
\begin{aligned}
u-\tilde{u}= & {\left[B^{-1}(\xi, \eta)-B^{-1}(\xi, \hat{\eta})\right] E_{r-1} \lambda_{r} \operatorname{sign}\left(\tilde{\xi}_{r}-\hat{\xi}_{r}\right) } \\
& -B^{-1}(\xi, \eta) e_{A}
\end{aligned}
$$

and consequently, from the convergence of $e_{A}$ and $(\eta-\hat{\eta})$ to zero, we have

$$
\lim _{t \rightarrow \infty}|u-\tilde{u}|=0
$$

\section{EXAMPLE}

In order to highlight the proposed method, we construct a simple multiple secure communication system based on the Qi's chaotic system in [9], which is described as follows:

$$
\left\{\begin{array}{l}
\dot{x}_{1}=a\left(x_{2}-x_{1}\right)+x_{2} x_{3} x_{4} \\
\dot{x}_{2}=b\left(x_{1}+x_{2}\right)-x_{1} x_{3} x_{4} \\
\dot{x}_{3}=-c x_{3}+x_{1} x_{2} x_{4} \\
\dot{x}_{4}=-d x_{4}+x_{1} x_{2} x_{3}
\end{array}\right.
$$

where $x_{i}(i=1: 5)$ are the state variables, and $a, b, c, d$ are all positive real constant parameters. Consider the following transmitter which is based on the chaotic system (17):

$$
\left\{\begin{array}{l}
\dot{x}_{1}=a\left(x_{2}-x_{1}\right)+x_{2} x_{3} x_{4}+m_{1} \\
\dot{x}_{2}=b\left(x_{1}+x_{2}\right)-x_{1} x_{3} x_{4} \\
\dot{x}_{3}=-c x_{3}+x_{1} x_{2} x_{4}+x_{3} x_{5} m_{2} \\
\dot{x}_{4}=-d x_{4}+x_{1} x_{2} x_{3}-x_{4} x_{5} m_{2} \\
\dot{x}_{5}=-10 x_{5}+x_{3} x_{4}
\end{array}\right.
$$

where $g_{1}=\left[\begin{array}{lllll}1 & 0 & 0 & 0 & 0\end{array}\right]^{T}$ and $g_{2}=$ 
and $g_{2}$ are involutive. Assume that $m_{1}$ and $m_{2}$ are small, that $m_{2}>0$, and that the following condition is satisfied

$$
m_{2}+\frac{d-c}{x_{5}}>0
$$

Suppose that the outputs are set as $y=\left[\begin{array}{ll}x_{1} & x_{2}\end{array}\right]^{T}$. The input channel vector fields $g_{1}$ and $g_{2}$ have been chosen such that the relative degree of the system is $r=3$. So, following the lines of the algorithm proposed in [1], set $£(x)=\operatorname{span}\left\{h_{1}, h_{2}, L_{f} h_{2}\right\}$. Since

$$
L_{f} h_{2}=b\left(x_{1}+x_{2}\right)-x_{1} x_{3} x_{4}=x_{3} x_{4} \bmod \left\{x_{1}, x_{2}\right\}
$$

one has $£(x)=\operatorname{span}\left\{x_{1}, x_{2}, x_{3} x_{4}\right\}$. Then let us calculate

$$
\Gamma=\left(\begin{array}{cc}
L_{g_{1}} h_{1} & L_{g_{2}} h_{1} \\
L_{g_{1}} L_{f} h_{2} & L_{g_{2}} L_{f} h_{2}
\end{array}\right)=\left(\begin{array}{cc}
1 & 0 \\
b-x_{3} x_{4} & 0
\end{array}\right) .
$$

One can choose $K=\left(b-x_{3} x_{4},-1\right)$ such that $K \Gamma=$ $(0,0)$.

Then the following fictitious output can be defined:

$$
\begin{aligned}
\bar{y} & =K\left[\begin{array}{c}
L_{f} h_{1} \\
L_{f}^{2} h_{2}
\end{array}\right]=\left(b-x_{3} x_{4}\right) \dot{y}_{1}-\ddot{y}_{2} \\
& =\left(x_{3}^{2}+x_{4}^{2}\right) \bmod £(x)
\end{aligned}
$$

because $\bar{y} \notin £(x)$, and $\bar{y}$ can be selected as a new fictitious output. Then, let us set

$$
y \triangleq\left[x_{1}, \quad x_{2}, \quad x_{3}^{2}+x_{4}^{2}\right]^{T} .
$$

With this new output $y$, we have $\bar{£}(x)=$ $\operatorname{span}\left\{x_{1}, x_{2}, x_{3} x_{4},\left(x_{3}^{2}+x_{4}^{2}\right)\right\}$, where $\operatorname{dim} \bar{£}(x)=4<5$. But due to the fact that $\left\{g_{1}, g_{2}\right\}$ is involutive, so according to Proposition 1, transmitter (18) can be transformed into the following form (7). Due to Proposition 2, we can recover both the state and the unknown messages in finite time. For this, let us design a sliding mode observer for system (18) as follows:

$$
\left\{\begin{array}{l}
\dot{\hat{x}}_{1}=a\left(x_{2}-x_{1}\right)+x_{2} \tilde{x}_{3} \tilde{x}_{4}+E_{1} \lambda_{1} \operatorname{sign}\left(x_{1}-\hat{x}_{1}\right) \\
\dot{\hat{x}}_{2}=b\left(x_{1}+x_{2}\right)+\lambda_{2} \operatorname{sign}\left(x_{2}-\hat{x}_{2}\right) \\
\frac{d\left(\hat{x}_{3} \hat{x}_{4}\right)}{d t}=-(c+d) \tilde{x}_{3} \tilde{x}_{4} \\
\quad+E_{2} \lambda_{3} \operatorname{sign}\left(\tilde{x}_{3} \tilde{x}_{4}-\hat{x}_{3} \hat{x}_{4}\right) \\
\frac{d\left(\hat{x}_{3}^{2}+\hat{x}_{4}^{2}\right)}{d t}=-2 c \tilde{x}_{3}^{2}-2 d \tilde{x}_{4}^{2}+4 x_{1} x_{2} \tilde{x}_{3} \tilde{x}_{4} \\
\quad+2 E_{3} \lambda_{4} \operatorname{sign}\left(\left(\tilde{x}_{3}^{2}+\tilde{x}_{4}^{2}\right)-\left(\hat{x}_{3}^{2}+\hat{x}_{4}^{2}\right)\right) \\
\dot{\hat{x}}_{5}=E_{3}\left(-10 \hat{x}_{5}+\tilde{x}_{3} \tilde{x}_{4}\right)
\end{array}\right.
$$

with

$$
\begin{aligned}
& \lambda_{i}>0, i=1, \ldots, 4 \\
& E_{1}= \begin{cases}1 & \text { if } x_{2}=\hat{x}_{2} \\
0 & \text { otherwise }\end{cases} \\
& E_{2}= \begin{cases}1 & \text { if } E_{1}=1 \text { and } x_{1}=\hat{x}_{1} \\
0 & \text { otherwise }\end{cases} \\
& E_{3}= \begin{cases}1 & \text { if } E_{2}=1 \text { and } \tilde{x}_{3} \tilde{x}_{4}=\hat{x}_{3} \hat{x}_{4} \\
0 & \text { otherwise }\end{cases} \\
& E_{4}= \begin{cases}1 & \text { if } E_{3}=1 \text { and } \hat{x}_{5}=x_{5} \\
0 & \text { otherwise }\end{cases}
\end{aligned}
$$

and with the auxiliary states:

$$
\begin{aligned}
\tilde{x}_{3} \tilde{x}_{4} & =-\frac{\lambda_{2} \operatorname{sign}\left(x_{2}-\hat{x}_{2}\right)}{x_{1}} \\
\tilde{x}_{3}^{2}+\tilde{x}_{4}^{2} & =\frac{E_{2} \lambda_{3} \operatorname{sign}\left(\tilde{x}_{3} \tilde{x}_{4}-\hat{x}_{3} \hat{x}_{4}\right)}{\hat{x}_{5} x_{1} x_{2}} .
\end{aligned}
$$

Obviously the submanifold of observability singularity $S$ is equal to $S=\left\{x_{1}=0\right\} \cup\left\{x_{1} x_{2}=0\right\}$.

Let us also define

$$
\begin{aligned}
& \tilde{m}_{1}=E_{2} \lambda_{1} \operatorname{sign}\left(x_{1}-\hat{x}_{1}\right) \\
& \tilde{m}_{2}=\frac{E_{5} \lambda_{4} \operatorname{sign}\left(\left(\tilde{x}_{3}^{2}+\tilde{x}_{4}^{2}\right)-\left(\hat{x}_{3}^{2}+\hat{x}_{4}^{2}\right)\right)}{\hat{x}_{5}\left(\tilde{x}_{3}^{2}-\tilde{x}_{4}^{2}\right)} .
\end{aligned}
$$

where

$$
E_{5}= \begin{cases}1 & \text { if } E_{4}=1 \text { and } \tilde{x}_{3}^{2}+\tilde{x}_{4}^{2}=\hat{x}_{3}^{2}+\hat{x}_{4}^{2} \\ 0 & \text { otherwise }\end{cases}
$$

The observation errors are defined by:

$$
\begin{aligned}
& e_{1}=x_{1}-\hat{x}_{1} \\
& e_{2}=x_{2}-\hat{x}_{2} \\
& e_{34}=x_{3} x_{4}-\hat{x}_{3} \hat{x}_{4} \\
& e_{3^{2}+4^{2}}=\left(x_{3}^{2}+x_{4}^{2}\right)-\left(\hat{x}_{3}^{2}+\hat{x}_{4}^{2}\right) \\
& e_{5}=x_{5}-\hat{x}_{5}
\end{aligned}
$$

From system (18), it can be computed that:

$$
\frac{d\left(x_{3} x_{4}\right)}{d t}=-(c+d) x_{3} x_{4}+x_{1} x_{2}\left(x_{3}^{2}+x_{4}^{2}\right)
$$

and

$$
\begin{aligned}
\frac{d\left(x_{3}^{2}+x_{4}^{2}\right)}{d t}= & -2 c x_{3}^{2}+4 x_{1} x_{2} x_{3} x_{4} \\
& -2 d x_{4}^{2}+2\left(x_{3}^{2}-x_{4}^{2}\right) m_{2} .
\end{aligned}
$$

Thus the dynamics of the observation error is given by

$$
\left\{\begin{array}{c}
\dot{e}_{1}=x_{2}\left(x_{3} x_{4}-\tilde{x}_{3} \tilde{x}_{4}\right)+m_{1}-E_{1} \lambda_{1} \operatorname{sign}\left(e_{1}\right) \\
\dot{e}_{2}=-x_{1} x_{3} x_{4}-\lambda_{2} \operatorname{sign}\left(e_{2}\right) \\
\dot{e}_{34}=-(c+d)\left(x_{3} x_{4}-\tilde{x}_{3} \tilde{x}_{4}\right)+x_{1} x_{2}\left(x_{3}^{2}+x_{4}^{2}\right) \\
\quad-E_{2} \lambda_{3} \operatorname{sign}\left(\tilde{x}_{3} \tilde{x}_{4}-\hat{x}_{3} \hat{x}_{4}\right) \\
\dot{e}_{3^{2}+4^{2}}=-2 c\left(x_{3}^{2}-\tilde{x}_{3}^{2}\right)-2 d\left(x_{4}^{2}-\tilde{x}_{4}^{2}\right) \\
\quad+4 x_{1} x_{2}\left(x_{3} x_{4}-\tilde{x}_{3} \tilde{x}_{4}\right)+2\left(x_{3}^{2}-x_{4}^{2}\right) m_{2} \\
\quad-2 E_{3} \lambda_{4} \operatorname{sign}\left(\left(\tilde{x}_{3}^{2}+\tilde{x}_{4}^{2}\right)-\left(\hat{x}_{3}^{2}+\hat{x}_{4}^{2}\right)\right) \\
\dot{e}_{5}=-10 x_{5}+x_{3} x_{4}-E_{3}\left(-10 \hat{x}_{5}+\tilde{x}_{3} \tilde{x}_{4}\right)
\end{array}\right.
$$

The convergence of the sliding mode observer has been proved in the previous section. But for this example, it should note that the possibility to estimate $m_{2}$ requires the knowledge of $\tilde{x}_{3}^{2}$ and $\tilde{x}_{4}^{2}$, which can be computed in the following way after the successful estimations of $\hat{x}_{5}, \tilde{x}_{3} \tilde{x}_{4}$ and $\left(\tilde{x}_{3}^{2}+\tilde{x}_{4}^{2}\right)$.

The convergence of $\tilde{x}_{3} \tilde{x}_{4}$ and $\left(\tilde{x}_{3}^{2}+\tilde{x}_{4}^{2}\right)$ can be easily obtained. Moreover as $x_{5}$ is uniformly exponentially detectable, we can detect $\hat{x}_{5}$. Indeed, we choose $V\left(e_{5}\right)=\frac{e_{5}^{2}}{2}$, then $\dot{V}\left(e_{5}\right)=-10 e_{5}^{2}<0$, which implies that the Hypothesis 1 is satisfied with $K_{0}=10$, and we obtain $\lim _{t \rightarrow+\infty}\left|x_{5}-\hat{x}_{5}\right|=0$. 
Then we can define: $\tilde{x}_{3} \tilde{x}_{4}=A$ and $\tilde{x}_{3}^{2}+\tilde{x}_{4}^{2}=B$, obviously there are two groups of solutions:

$$
\begin{aligned}
& S_{1}:\left\{\begin{array}{l}
\tilde{x}_{3_{1}}^{2}=\frac{B+\sqrt{B^{2}-4 A^{2}}}{2} \\
\tilde{x}_{4_{1}}^{2}=\frac{B-\sqrt{B^{2}-4 A^{2}}}{2}
\end{array}\right. \text { and } \\
& S_{2}:\left\{\begin{array}{l}
\tilde{x}_{3_{2}}^{2}=\frac{B-\sqrt{B^{2}-4 A^{2}}}{2} \\
\tilde{x}_{4_{2}}^{2}=\frac{B+\sqrt{B^{2}-4 A^{2}}}{2}
\end{array}\right.
\end{aligned}
$$

Suppose that $S_{1}$ is the correct solution. From (25), the confidential message can be recovered correctly as follows:

$$
-c \tilde{x}_{3_{1}}^{2}-d \tilde{x}_{4_{1}}^{2}+\left(\tilde{x}_{3_{1}}^{2}-\tilde{x}_{4_{1}}^{2}\right) \hat{x}_{5} m_{2_{1}}=-2 x_{1} x_{2} \tilde{x}_{3} \tilde{x}_{4} \triangleq C .
$$

In this case, one has for $S_{2}$ :

$$
-c \tilde{x}_{3_{2}}^{2}-d \tilde{x}_{4_{2}}^{2}+\left(\tilde{x}_{3_{2}}^{2}-\tilde{x}_{4_{2}}^{2}\right) \hat{x}_{5} m_{2_{2}}=C .
$$

Using equations (27) and (28), one has:

$$
m_{2_{2}}=\frac{\left[\begin{array}{l}
-c \tilde{x}_{3_{1}}^{2}-d \tilde{x}_{4_{1}}^{2}+\left(\tilde{x}_{3_{1}}^{2}-\tilde{x}_{4_{1}}^{2}\right) \hat{x}_{5} m_{2_{1}} \\
+c \tilde{x}_{3_{2}}^{2}+d \tilde{x}_{4_{2}}^{2}
\end{array}\right]}{\left(\tilde{x}_{3_{2}}^{2}-\tilde{x}_{4_{2}}^{2}\right) \hat{x}_{5}}
$$

Note that $\tilde{x}_{3_{1}}^{2}=\tilde{x}_{4_{2}}^{2}$ and $\tilde{x}_{3_{2}}^{2}=\tilde{x}_{4_{1}}^{2}$. So this equation becomes

$$
\begin{aligned}
m_{2_{2}} & =\frac{\left[\begin{array}{l}
-c \tilde{x}_{3_{1}}^{2}-d \tilde{x}_{4_{1}}^{2}+\left(\tilde{x}_{3_{1}}^{2}-\tilde{x}_{4_{1}}^{2}\right) \hat{x}_{5} m_{2_{1}} \\
+c \tilde{x}_{4_{1}}^{2}+d \tilde{x}_{3_{1}}^{2}
\end{array}\right]}{\left(\tilde{x}_{4_{1}}^{2}-\tilde{x}_{3_{1}}^{2}\right) \hat{x}_{5}} \\
& =\frac{c-d}{\hat{x}_{5}}-m_{2_{1}}
\end{aligned}
$$

If $m_{2_{1}}$ is the correct solution, then $m_{2_{2}}<0$ according to equation (19) and this excludes the solution $m_{2_{2}}$. Following this way, the correct solution corresponding to $\tilde{x}_{3}^{2}$ and $\tilde{x}_{4}^{2}$ can be found.

Since $\tilde{x}_{3}^{2}$ and $\tilde{x}_{4}^{2}$ have been estimated, one has:

$$
\dot{e}_{3^{2}+4^{2}}=2 x_{5}\left(x_{3}^{2}-x_{4}^{2}\right) m_{2}-2 E_{3} \lambda_{4} \operatorname{sign}\left(e_{3^{2}+4^{2}}\right)
$$

Thus, tuning $\lambda_{4}>\left|x_{5}\left(x_{3}^{2}-x_{4}^{2}\right) m_{2}\right|_{\max }$ ensures that

$$
\lim _{t \rightarrow+\infty}\left|x_{5}\left(x_{3}^{2}-x_{4}^{2}\right) m_{2}-\lambda_{4} \operatorname{sign}\left(e_{3^{2}+4^{2}}\right)\right|=0 .
$$

Then the relation (24) leads to the estimation of the second confidential message:

$$
\begin{aligned}
& \lim _{t \rightarrow+\infty}\left|\tilde{m}_{2}-m_{2}\right| \\
= & \lim _{t \rightarrow+\infty}\left|\frac{E_{4} \lambda_{4} \operatorname{sign}\left(e_{3^{2}+4^{2}}\right)}{\hat{x}_{5}\left(\tilde{x}_{3}^{2}-\tilde{x}_{4}^{2}\right)}-\frac{E_{4} \lambda_{4} \operatorname{sign}\left(e_{3^{2}+4^{2}}\right)}{x_{5}\left(\tilde{x}_{3}^{2}-\tilde{x}_{4}^{2}\right)}\right| \\
= & 0 .
\end{aligned}
$$

Figure 1 exhibits the states of the transmitter and those of the receiver. Figure 2 illustrates the original messages and their estimation. Figure 1 shows that the states of the receiver converge fast to those of the transmitter. It can be seen in Figure 2 that, once the state is estimated, the confidential messages are well reconstructed.

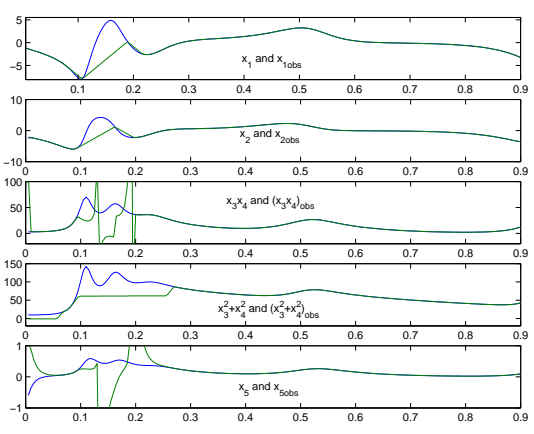

Fig. 1. States observation for transmitter and the receiver
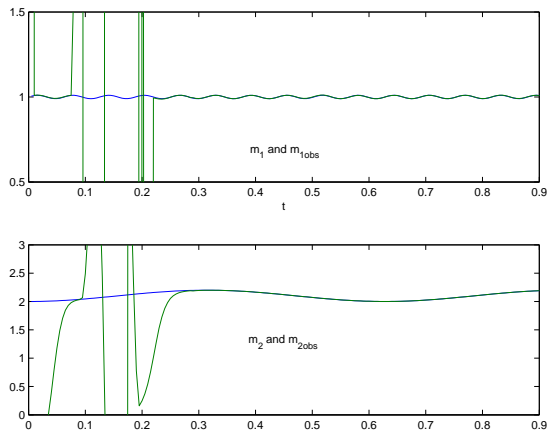

Fig. 2. Message observation for transmitter and the receiver

\section{CONCLUSION}

The topic of this paper is to apply the the algorithm presented by [1] into multi data secure communication. Based on this algorithm, we propose a new scheme, under the condition on zero dynamics, to solve the Left Invertibility Problem. Moreover, an illustrated example based on one Qi's chaotic system is established in order to highlight the proposed method.

\section{REFERENCES}

[1] Barbot J.P, Boutat D. and Floquet T. "A new observation algorithm for nonlinear system with unknown inputs". IEEE CDC-ECC., seville, Dec 2005.

[2] Darouach M., Zasadzinski M. and Xu S.J., Full-order Observers for linear systems with unknown inputs. IEEE Tans. on Auto. Contr., Vol. 39(3), pp 606-609, 1994.

[3] Floquet, T, Barbot, J.P., "A slidin mode approach of unknown input observers for linear systems". IEEE CDC., pp 1724-1729, 2004.

[4] Hautus, M.L.J, Strong detectability and observers. Linear algebra and its applications., vol. 50, pp 353-368.

[5] Hou, M. , Mülle, P.C., Disturbance decoupled observer design: A unified viewpoint. IEEE Trans. on Auto. Contr., vol. 39(6), pp 632-635, 1994.

[6] Isidori A., Nonlinear control systems., 3rd edition, Springer, Berlin, 1995.

[7] Nijmeijer H. and Mareels I. M. Y. An observer looks at synchronization. IEEE Trans. on Circuits and Systems-1: Fundamental theory and Applications., vol. 44(10), pp 882-891, 1997.

[8] Pecora L. M. and Carroll T. L. Synchronization in chaotic systems. Physical Review Letters. vol. 64, pp 821-824, 1990.

[9] Qi G.Y., Du S.Z., Chen G.R. et al. On a four-dimensional chaotic system. Chaos, Solitons and Fractals., vol. 23, pp 2005.

[10] Singh S.N., A modified algorithm for invertibility in nonlinear system, IEEE Trans. on Auto. Contr., vol. 26(2), pp 595-598, 1981.

[11] Xiong. Y, Saif, M, Sliding mode observer for nonlinear uncertain systems. IEEE Trans. on Auto. Contr., vol. 46, pp 2012-2017, 2001. 